\title{
Adipose-derived stem cells alleviate radiation-induced dermatitis by suppressing apoptosis and downregulating cathepsin $\mathrm{F}$ expression
}

Chaoling Yao ${ }^{1}$, Yue Zhou ${ }^{2}$, Hui Wang ${ }^{2}$, Feiyan Deng ${ }^{3}$, Yongyi Chen ${ }^{4}$, Xiaomei Zhu ${ }^{4}$ Yu Kong ${ }^{5}$, Lijun Pan ${ }^{5}$, Lei Xue Xiao Zhou ${ }^{1}$, Chunmeng Shi $^{7}$ and Xiaowu Sheng ${ }^{1 *}$

\begin{abstract}
Background: Radiation-induced dermatitis is a serious side effect of radiotherapy, and very few effective treatments are currently available for this condition. We previously demonstrated that apoptosis is an important feature of radiation-induced dermatitis and adipose-derived stem cells (ADSCs) are one of the most promising types of stem cells that have a protective effect on acute radiation-induced dermatitis. Cathepsin F (CTSF) is a recently discovered protein that plays an important role in apoptosis. In this study, we investigated whether ADSCs affect chronic radiation-induced dermatitis, and the underlying mechanisms involved.
\end{abstract}

Methods: ADSCs were isolated from male Sprague-Dawley (SD) rats and characterized. For in vivo studies, rats were randomly divided into control and ADSC-treated groups, and cultured ADSCs were transplanted into radiationinduced dermatitis model rats. The effects of ADSC transplantation were determined by skin damage scoring, histopathological analysis, electron microscopy, immunohistochemical staining, and western blotting analysis of apoptosis-related proteins. To evaluate the effects of ADSCs in vitro, radiation-induced apoptotic cells were treated with ADSC culture supernatant, and apoptosis-related protein expression was investigated by TUNEL staining, flow cytometry, and western blotting.

Results: In the in vivo studies, skin damage, inflammation, fibrosis, and apoptosis were reduced and hair follicle and sebaceous gland regeneration were enhanced in the ADSC group compared with the control group. Further, CTSF and downstream pro-apoptotic proteins (Bid, BAX, and caspase 9) were downregulated, while anti-apoptotic proteins (BCl-2 and $\mathrm{BCl}-\mathrm{XL}$ ) were upregulated. In vitro, ADSCs markedly attenuated radiation-induced apoptosis, downregulated CTSF and downstream pro-apoptotic proteins, and upregulated anti-apoptotic proteins.

Conclusion: ADSCs protect against radiation-induced dermatitis by exerting an anti-apoptotic effect through inhibition of CTSF expression. ADSCS may be a good therapeutic candidate to prevent the development of radiation-induced dermatitis.

Keywords: Adipose-derived stem cells, Apoptosis, Cathepsin F, Radiation-induced dermatitis

\footnotetext{
*Correspondence: shengxiaowu789@163.com

'Department of Head and Neck Surgery, Central Laboratory, Hunan Cancer Hospital and The Affiliated Cancer Hospital of Xiangya School of Medicine, Tongzipo Road 283, Changsha 410013, Hunan Province, China

Full list of author information is available at the end of the article
} 


\section{Background}

Radiation-induced dermatitis is a serious side effect of radiotherapy, up to $95 \%$ of patients who undergo radiotherapy develop dermatitis. Acute radiation-induced dermatitis occurs within a few days after radiotherapy and is characterized by erythema and desquamation [1, 2]. Chronic radiation-induced dermatitis usually occurs more than 90 days after radiotherapy and is characterized by fibrosis and thickening of the dermis [3, 4]. Importantly, radiation-induced dermatitis may limit the radiation dose delivered and even necessitate treatment termination. Currently, this condition is treated using drugs, wound dressing, and even surgical treatment [1, 5]. However, these interventions have various limitations, such as poor efficacy or high risk; therefore, new effective treatments are urgently needed.

In our previous study, we established a rat model of radiation-induced dermatitis and found that apoptosis is an important feature of this condition [6]. Apoptosis is a major route of radiation-induced cell death. Radiation can directly break chemical bonds in the DNA backbone, creating single- and double-strand breaks, thus causing DNA damage in irradiated cells. Double-strand breaks in normal cells activate ataxia telangiectasiamutated and DNA-dependent protein kinase [7], which phosphorylate histone $\mathrm{H} 2 \mathrm{AX}$ and P53-binding protein 53BP1 and guide cells toward repair or ultimately induce cell cycle arrest and apoptosis [8]. Moreover, ionizing radiation interacts with water molecules to form reactive oxygen species, such as superoxide, hydrogen peroxide, and hydroxyl radicals [9]. Reactive oxygen species activate several signaling pathways, including p53-mediated apoptosis, mitogen-activated protein kinase signaling, nuclear factor E2-related factor 2 signaling, hypoxiainducible factor- $1 \alpha$ signaling, and nuclear factor $\mathrm{kB}$ signaling [10], and induce cell injury by damaging proteins and nucleic acids. Injured cells release various inflammatory chemokines and cytokines and attract inflammatory cells to the site of injury, thus facilitating the release of more proinflammatory cytokines, such as tumor necrosis factor- $\alpha$, interleukin (IL)-1, interferon- $\gamma$, and IL-6 [11]. Increased levels of these proinflammatory cytokines activate pro-apoptotic signals and induce apoptosis [12].

Cathepsin F (CTSF) is a recently described papain-like cysteine protease that plays an important role in the apoptotic process [13]. CTSF expression is downregulated in gastric cancer cells and tissues, and CTSF knockdown significantly suppressed gastric cancer cell apoptosis [14]. KEGG pathway database analysis revealed that CTSF reduces Bcl-2 in the apoptotic pathway [14]. Janic et al. showed that CTSF knockdown induced leukemia development [15]. However, whether CTSF is involved in the pathogenesis of radiation-induced dermatitis is unclear.
Adipose-derived stem cells (ADSCs) are a type of mesenchymal stem cells that are derived from adipose tissue. They can readily adhere to plastic culture flasks, are easily expanded in vitro, and are considered to have broad clinical application potential. ADSCs were first isolated by Zuk et al. [16]. ADSCs can differentiate into multiple cell lineages and secrete various paracrine factors. For example, they secrete hepatocyte growth factor, IL-12, and superoxide dismutase (an enzyme with anti-oxidant effects); vascular-derived and platelet-derived growth factors to exert pro-angiogenic effects; stromal-derived factor-1 to recruit non-injured stem cells to the injury site; and insulin-like growth factor-1 to promote hair regeneration [17]. Furthermore, exosomes from ADSCs can reduce neuronal cell apoptosis [18]. ADSCs have been shown to exhibit anti-inflammatory effects in a bleomycin-induced interstitial pneumonia mouse model [19] and to attenuate pulmonary fibrosis of silicosis via anti-inflammatory and anti-apoptotic effects [20]. We have previously demonstrated that ADSCs have a protective effect on acute radiation-induced dermatitis [21]. However, whether they have a similar effect on chronic radiation-induced dermatitis and the underlying mechanisms remain unclear.

Therefore, in this study, we evaluated the effects of ADSCs on acute and chronic radiation-induced dermatitis, and we preliminarily analyzed their underlying mechanism.

\section{Methods \\ Experimental animals and wound assessment after irradiation}

Sprague-Dawley (SD) rats weighing 220-250 g were obtained from Hunan SJA Laboratory Animal Co., Ltd. (Hunan, China). All researchers who performed the animal operations received training in animal experiments from Central South University. Ethics approval was obtained from the Animal Ethics Committee of Hunan Cancer Hospital and the Affiliated Cancer Hospital of Xiangya School of Medicine, Central South University. To establish the radiation-induced dermatitis model, SD rats were irradiated as described previously [6]. The rats were anesthetized by pentobarbital sodium (5\%, $50 \mathrm{mg} /$ $\mathrm{kg}$ ) injection. Forty-eight female SD rats were randomly divided into control and ADSC groups $(n=24$ per group). Rats in the control group received 90 Gy of irradiation and injected with PBS, whereas those in the ADSC group were irradiated with 90 Gy and then injected with $10^{7}$ ADSCs within $24 \mathrm{~h}$. The degree of skin tissue toxicity after irradiation was scored independently by two individuals who were blinded to the groups, according to previous reports [22, 23]. Wound scores and pictures were recorded weekly until 12 weeks after the operation. 


\section{Isolation, differentiation, and characterization of rat ADSCs}

Inguinal adipose tissue was harvested from male rats after they were euthanized. Subcutaneous visible blood vessels were removed, and the tissue was rinsed with phosphate-buffered saline (PBS) (Hyclone, Logan, UT, USA), cut into pieces, and subjected to enzymatic digestion with $1 \mu \mathrm{g} / \mathrm{ml}$ type I collagenase (Invitrogen, Carlsbad, CA, USA) in a constant-temperature water bath at $37{ }^{\circ} \mathrm{C}$ for $60 \mathrm{~min}$. After neutralization of the collagenase using cell culture medium, the dispersed tissue was centrifuged at $1000 \times g$ at $25^{\circ} \mathrm{C}$ for $10 \mathrm{~min}$. The floating fat was aspirated, and the cell suspension was filtered through a $70-\mu \mathrm{m}$ screen mesh to remove tissue fragments. The filtrate was then centrifuged at $1000 \times g$ for $10 \mathrm{~min}$, the supernatant was discarded, and the cells were collected and cultured in Dulbecco's modified Eagle's medium (DMEM, Gibco, Grand Island, NY, USA) supplemented with $10 \%$ fetal bovine serum (Gibco, Carlsbad, CA, USA) and $1 \%$ penicillin/streptomycin (Gibco, Grand Island, NY, USA) at $37^{\circ} \mathrm{C}$ in a $5 \% \mathrm{CO}_{2}$ atmosphere. For cell differentiation and identification, ADSCs from passage 3 were used. In vitro differentiation of ADSCs was induced using adipogenic, chondrogenic, and osteogenic differentiation media (Cyagen, Chicago, IL, USA) in six-well plates. ADSC differentiation into adipocytes was induced for 20 days, and differentiation into chondrocytes and osteoblasts for 21 days. Differentiated cells were fixed and stained with oil red $\mathrm{O}$, alcian blue, or alizarin red and observed under a microscope (Carl Zeiss, Oberkochen, Germany). The expression of ADSC immunomarkers, including CD10, CD34, CD45, CD73, CD90, and CD105 (Abcam, Cambridge, UK), was evaluated by flow cytometry.

\section{Cell culture, irradiation, and treatments}

Human immortalized keratinocytes (HaCaT; Wuhan Yipu Biological Technology Co., Ltd., Wuhan, China) and normal human oral squamous epithelial cells (NOK; Shanghai Binsui Biological Technology Co., Ltd., Shanghai, China) were cultured in DMEM supplemented with $10 \%$ fetal bovine serum and $1 \%$ streptomycin/penicillin. $\mathrm{HaCaT}$ and NOK cells were irradiated with a linear accelerator (Varian Rapid ARC, Palo Alto, CA, USA) at doses of 20 and 5 Gy, respectively. The dose rate was $600 \mathrm{Mu} / \mathrm{min}$. ADSC culture supernatant was collected and centrifuged at $1000 \times g$ for $5 \mathrm{~min}$ to remove cell debris. Irradiated cells were immediately treated with ADSC culture supernatant. Irradiated cells treated with normal culture medium served as a control. The medium was changed every $24 \mathrm{~h}$. Cells were collected $72 \mathrm{~h}$ after irradiation for apoptosis and for western blotting assays.

\section{Histological staining}

Skin tissues were fixed in $4 \%$ paraformaldehyde, paraffin-embedded, and sectioned at $4-\mu \mathrm{m}$ thickness. The sections were routinely stained with hematoxylin and eosin (Servicebio, Wuhan, China) and Masson's trichrome (Servicebio, Wuhan, China). The cells were analyzed and imaged under an optical microscope (Carl Zeiss, Oberkochen, German).

\section{Hydroxyproline content assay}

To quantify collagen in skin tissues, the hydroxyproline content was measured using a hydroxyproline assay kit (Jiancheng Bioengineering Institute, Nanjing, China) following the manufacturer's protocol, as previously reported [6]. The hydroxyproline content was normalized to wet tissue weight.

\section{Immunohistochemical staining}

Immunohistochemical staining was performed as described previously [6]. BAX polyclonal antibody (1:200; $50599-2-\mathrm{Ig}), \mathrm{Bcl} 2$ polyclonal antibody (1:200; 26593-1AP), and CTSF polyclonal antibody (1:200; 11055-1-AP) were all purchased from ProteinTech (Chicago, IL, USA). To quantify protein levels, the average optical density (OD) value of positive staining was determined using the Image-Pro Plus software.

\section{Electron microscopy}

Skin tissues were fixed with $2.5 \%$ glutaraldehyde in phosphate buffer and sliced into $50-\mu \mathrm{m}$ sections. The ultrastructure of skin cells and organelles was evaluated using an electron microscope (FEI, Hillsboro, OR, USA).

\section{Terminal deoxynucleotidyl transferase dUTP neck-end labeling (TUNEL) staining}

TUNEL staining was performed using a TUNEL Apoptosis Assay Kit (G1501; Servicebio, Wuhan, China), as described previously [6]. TUNEL $^{+}$cells were counted manually under an inverted fluorescence microscope (Carl Zeiss, Oberkochen, German).

\section{Flow-cytometric detection of apoptosis}

Apoptosis of $\mathrm{NOK}$ and $\mathrm{HaCaT}$ cells after irradiation and treatment with ADSC culture supernatant was detected using an Annexin V-APC/PI apoptosis detection kit (KG, Nanjing). Briefly, cells were harvested, washed twice with PBS, and resuspended in binding buffer. Annexin V-APC and PI were added, and the cells were incubated at room temperature in the dark for $10 \mathrm{~min}$ and then analyzed within an hour using a flow cytometer (Beckman, Hialeah, FL, USA). 
Table 1 SiRNAs used in this study

\begin{tabular}{lll}
\hline Homo sapiens CTSF & Sense $\mathbf{( 5 ^ { \prime } - \mathbf { 3 } ^ { \prime } )}$ & Antisense (5'-3') \\
\hline CTSF-001 & GGCUCAGCCAUGAUUUCUUTT & AAGAAAUCAUGGCUGAGCCTT \\
CTSF-002 & CCAUCAAUGCCUUUGGCAUTT & AUGCCAAAGGCAUUGAUGGTT \\
Negative control & UUCUCCGAACGUGUCACGUTT & ACGUGACACGUUCGGAGAATT \\
\hline
\end{tabular}

\section{Small interfering (si)RNA transfection}

SiRNAs targeting CTSF and negative control siRNA (Table 1) were synthesized at GenePharma (Shanghai, China). HaCaT cells were transfected using GPtransfect-Mate Transfection Reagent (Gene Pharma, Shanghai, China) according to the manufacturer's protocol.

\section{Western blotting}

Proteins were extracted from skin tissues and cells using RIPA lysis buffer and were quantified using the bicinchoninic acid method. The proteins were subjected to sodium dodecyl sulfate-polyacrylamide gel electrophoresis and transferred to nitrocellulose membranes. The membranes were blocked in 5\% nonfat milk at room temperature for $1 \mathrm{~h}$. Next, they were incubated with antibodies against CTSF $(0.1 \mu \mathrm{g} / \mathrm{mL} ; \mathrm{R} \& \mathrm{D}$, AF2075, Minneapolis, MN, USA), Bid (1:1000; 10988-1-AP, ProteinTech, USA), BAX (1:5000; 50599-2-Ig, ProteinTech, USA), Bcl-2 (1:500; 26593-1-AP, ProteinTech, USA), Bcl-XL (1:1000; 10783-1-AP, ProteinTech, USA), caspase 9 (1:300; 10380-1-AP, ProteinTech, USA), and GAPDH (1:5000; 10494-1-AP, ProteinTech, USA) at $4{ }^{\circ} \mathrm{C}$ overnight. After washing, the membranes were incubated with horseradish peroxidase-labeled goat anti-rabbit IgG (1:6000; SA00001-2, ProteinTech, USA) for $2 \mathrm{~h}$. The blots were developed using enhanced chemiluminescence, and band densities were analyzed using the Quantity One software (Bio-Rad, Hercules, CA, USA).

\section{Statistical analysis}

All data are presented as the mean \pm standard error of the mean and were analyzed using SPSS (version 13.0; SPSS, Chicago, IL, USA). Differences between groups were analyzed using Student's $t$-test; $p<0.05$ was considered statistically significant.

\section{Results}

\section{Isolation and characterization of ADSCs}

ADSCs were isolated from groin adipose tissues of male rats. Cultured ADSCs had a spindle shape (Fig. 1A) and adipogenic (Fig. 1B, C), osteogenic (Fig. 1D, E), and chondrogenic (Fig. 1F) differentiation potential. The ADSCs were analyzed for the expression of specific surface markers; they were strongly positive for CD10 $(97.02 \% \pm 0.73 \%)$, CD73 (96.67\% $\pm 1.38 \%)$, CD90 $(97.24 \% \pm 1.40 \%)$, and CD105 (96.96\% $\pm 1.46 \%)$, but negative for CD34 $(0.36 \% \pm 0.14 \%)$ and CD45 $(0.28 \% \pm$ 0.08\%) (Fig. 1G).

\section{ADSCs alleviate radiation-induced acute skin reaction}

In the control group, visible skin damage was detected from 5 days post-irradiation. At 2 weeks postirradiation, the skin showed remarkable erythema, desquamation, and hair loss. At 3 weeks post-irradiation, large ulcers, open wounds, and full-thickness skin loss were observed. At 8 weeks post-irradiation, most of the visible skin damage was repaired by scar tissue. In the ADSC group, visible skin damage was detected at the same time points as in the control group. At 2 weeks post-irradiation, the skin showed erythema and desquamation, but less hair loss than in the control group. At 3, 4, and 5 weeks post-irradiation, the skin exhibited smaller ulcers and open wounds than in the control group (Fig. 2A, indicated by a red dotted line). Most of the visible skin damage was repaired by scar tissue at 4 weeks post-irradiation, which was earlier than in the control group (Fig. 2A). Skin injury scores during the observation period are shown in Fig. 2B; the scores were lower in the ADSC group than in the control group from 3 to 5 weeks post-irradiation (Fig. 2B, green dotted line).

\section{ADSCs alleviate acute and chronic radiation-induced dermatitis}

Skin tissues from rats of the control and ADSC groups were collected at 4 and 12 weeks post-irradiation for histological analysis (Fig. 3A-D). In the control group, at 4 weeks post-irradiation, epidermal loss (Fig. 3A (1)), epidermal thickening (Fig. 3A2, red line), lymphocyte infiltration (Fig. 3A $(3,4)$, green circle), few blood vessels (Fig. 3A (2, 4), green arrow), and skin appendage loss (Fig. 3A (1), yellow curve) were observed. By contrast, at the same time point, the ADSC group showed no epidermal loss, less epidermal thickening (Fig. 3B (2), red line), low lymphocyte infiltration (Fig. 3B (3, 4), green circle), numerous blood vessels (Fig. 3B (3, 4), green arrow), and less skin appendage loss (Fig. 3B (1), yellow curve). At 12 weeks post-irradiation, in the control group, considerable dermal fibrosis with appendage loss (Fig. 3C (1), yellow curve), low lymphocyte infiltration (Fig. 3C (2), green circle), and few blood vessels (Fig. 3C $(2,4)$, green arrow) were observed. At the same time point, the ADSC group showed less dermal fibrosis than 


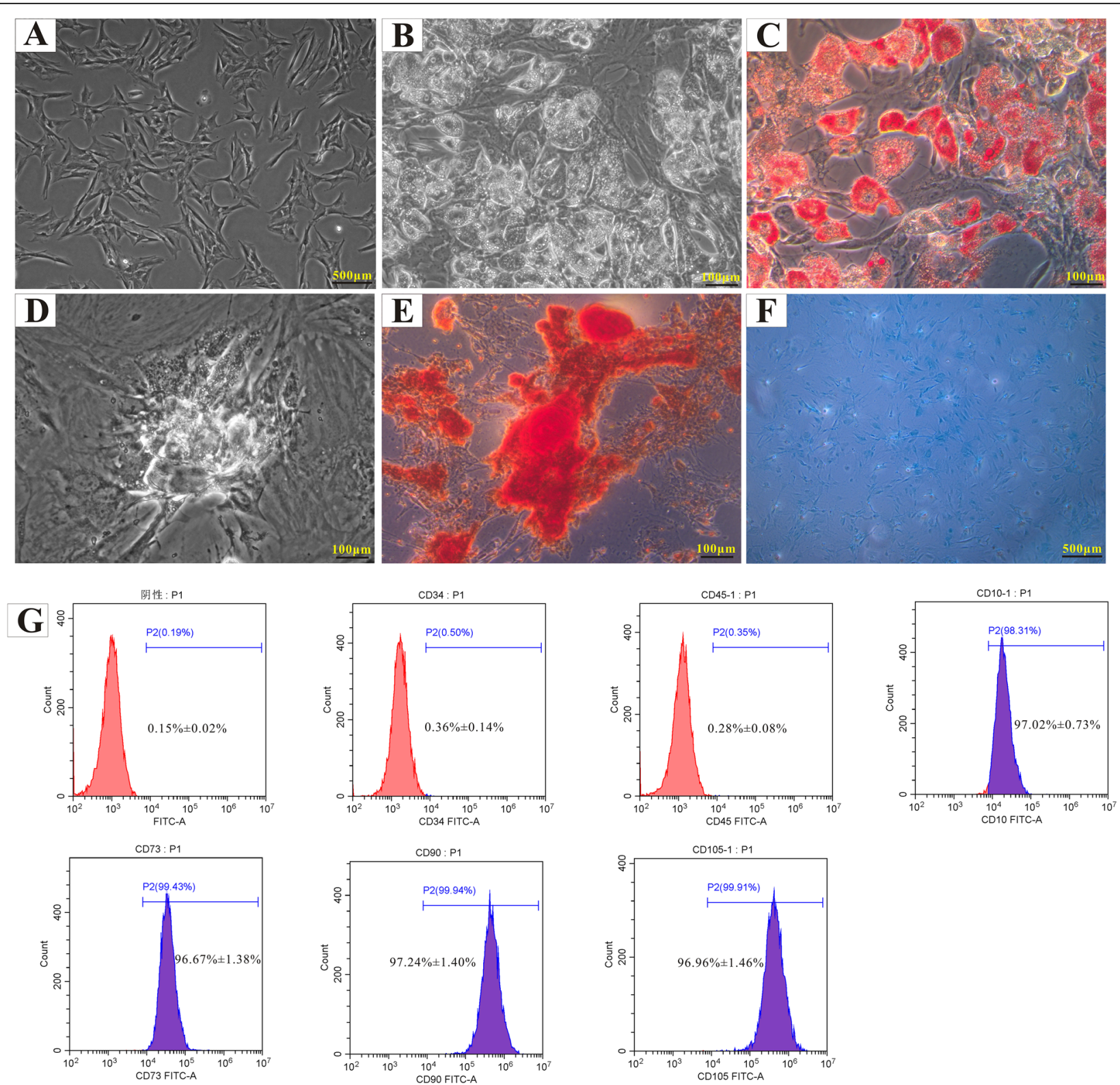

Fig. 1 Isolation and characterization of ADSCs. ADSCs isolated from male rat adipose tissue exhibited a typical spindle shape in culture (A). The multilineage differentiation potential of the ADSCs was investigated: adipogenesis was demonstrated by oil red O staining (B, C), osteogenesis by alizarin red $\mathbf{S}$ staining $(\mathbf{D}, \mathbf{E})$, and chondrogenesis by alcian blue staining $(\mathbf{F})$. The expression of ADSC surface markers was evaluated by flow cytometry $(\mathbf{G})$

the control group (Fig. 3D (1), yellow circle), and keratinization (Fig. 3D (3)).

Histological analysis of tissue sections confirmed that epidermal thickness was significantly lower in the ADSC group than in the control group at both 4 and 12 weeks post-irradiation (Fig. 3E). Similarly, dermal thickness was significantly lower in the ADSC group than in the control group at both 4 and 12 weeks post-irradiation (Fig. 3E). Hair follicle density was higher in the ADSC group than in the control group at both 4 and 12 weeks post-irradiation compared (Fig. 3E). Migrating hair follicle density was slightly, albeit not significantly, higher in the ADSC group than in the control group at 4 weeks post-irradiation (Fig. 3E) and was significantly lower at 12 weeks post-irradiation in the ADSC group than in the control group (Fig. 3E). Sebaceous gland density was slightly, albeit not significantly, higher in the ADSC group than in the control group at 4 weeks postirradiation (Fig. 3E) and was significantly lower at 12 weeks post-irradiation in the ADSC group than in the control group (Fig. 3E). The number of lymphocytes per 400× field was significantly lower in the ADSCs rats than 


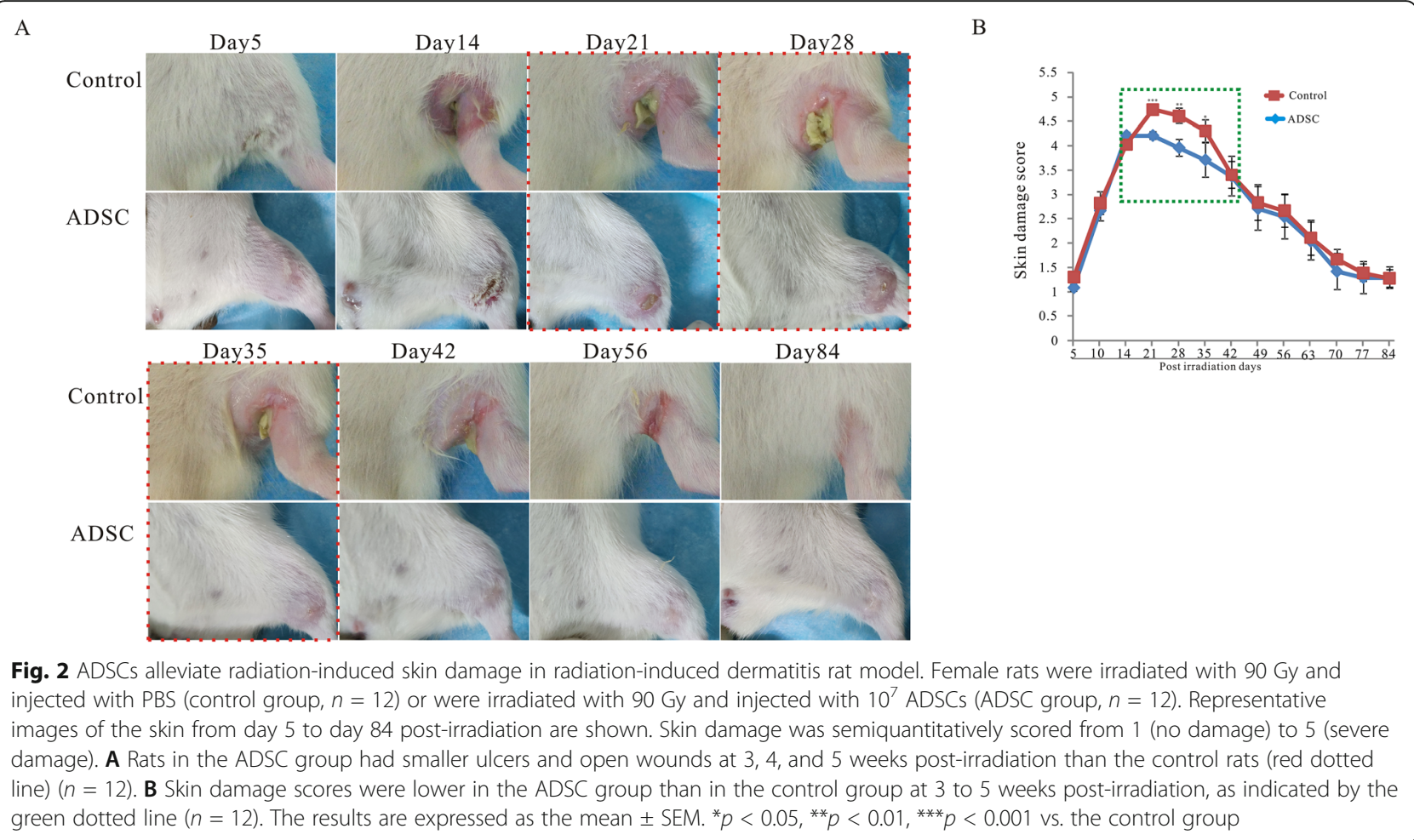

in the control rats at 4 and 12 weeks post-irradiation (Fig. 3E).

Fibrosis was analyzed using Masson's trichrome staining (Fig. 4A-D) and by measuring the hydroxyproline content in skin tissues. The collagen fiber percentage was lower in the ADSC group than in the control group at both 4 and 12 weeks post-irradiation (Fig. 4E). The hydroxyproline content of skins of rats of the ADSC group was significantly lower than that in control rats at 4 and 12 weeks post-irradiation (Fig. 4F).

\section{ADSCs inhibit apoptosis in irradiated skin tissue}

Transmission electron microscopy showed squamous cells with irregular desmosomes (Fig. 5A, B, yellow curve), damaged mitochondria with a loss of cristae (Fig. $5 \mathrm{~B}, \mathrm{C}$, green arrow), nuclear pyknosis and dissolution (Fig. 5D, E, indicated with "N"), and vascular endothelium swelling (Fig. 5F, red arrow) in skin tissues of rats of the control group at 4 weeks post-irradiation. However, in the ADSC group, at 4 weeks post-irradiation, squamous cells with regular desmosomes (Fig. 5G, large green curve), a large endoplasmic reticulum (Fig. 5J, small green curve), a well-formed autophagic vacuole (Fig. 5J, red circle), mitochondria with a few cristae left (Fig. 5I, blue arrow), regular nuclei (Fig. 5G-L, indicated with "N"), and a regularly shaped vascular endothelium (Fig. 5L, yellow arrow) were observed.

Representative fluorescence photomicrographs of TUNEL-positive apoptotic cells are shown in Fig. 6A.
The percentage of apoptotic cells was significantly lower in the ADSC group than in the control group at both 4 and 12 weeks post-irradiation (Fig. 6B). Representative immunohistochemical staining images are shown in Fig. 6C. The expression of the anti-apoptotic protein $\mathrm{Bcl}-2$ was significantly higher in the ADSC group than in the control group at both 4 and 12 weeks post-irradiation, whereas that of the pro-apoptotic protein BAX was markedly lower in rats of the ADSC group than in control rats at these two time points. Collectively, these findings suggested that ADSCs inhibit apoptosis in irradiated skin tissues in vivo.

\section{ADSCs inhibit apoptosis in irradiated epidermal cells}

Considering the remarkable apoptosis-inhibitory effect of ADSCs in vivo and that paracrine action is the main function of ADSCs, we further analyzed the effect of ADSCs on apoptosis in vitro. We first established radiation-induced apoptosis in $\mathrm{HaCaT}$ and NOK cells. Then, the irradiated cells were treated with ADSC culture supernatant. At $72 \mathrm{~h}$ postirradiation, apoptosis was analyzed by flow cytometry and TUNEL staining (Fig. 7A, B). Flow-cytometric data showed that the proportions of NOK cells in early, late, and total apoptosis were lower in the ADSC culture supernatant-treated group than in the control group (Fig. 7A). Similarly, the proportions of $\mathrm{HaCaT}$ cells in late and total apoptosis were lower in the supernatant-treated group than in the control 

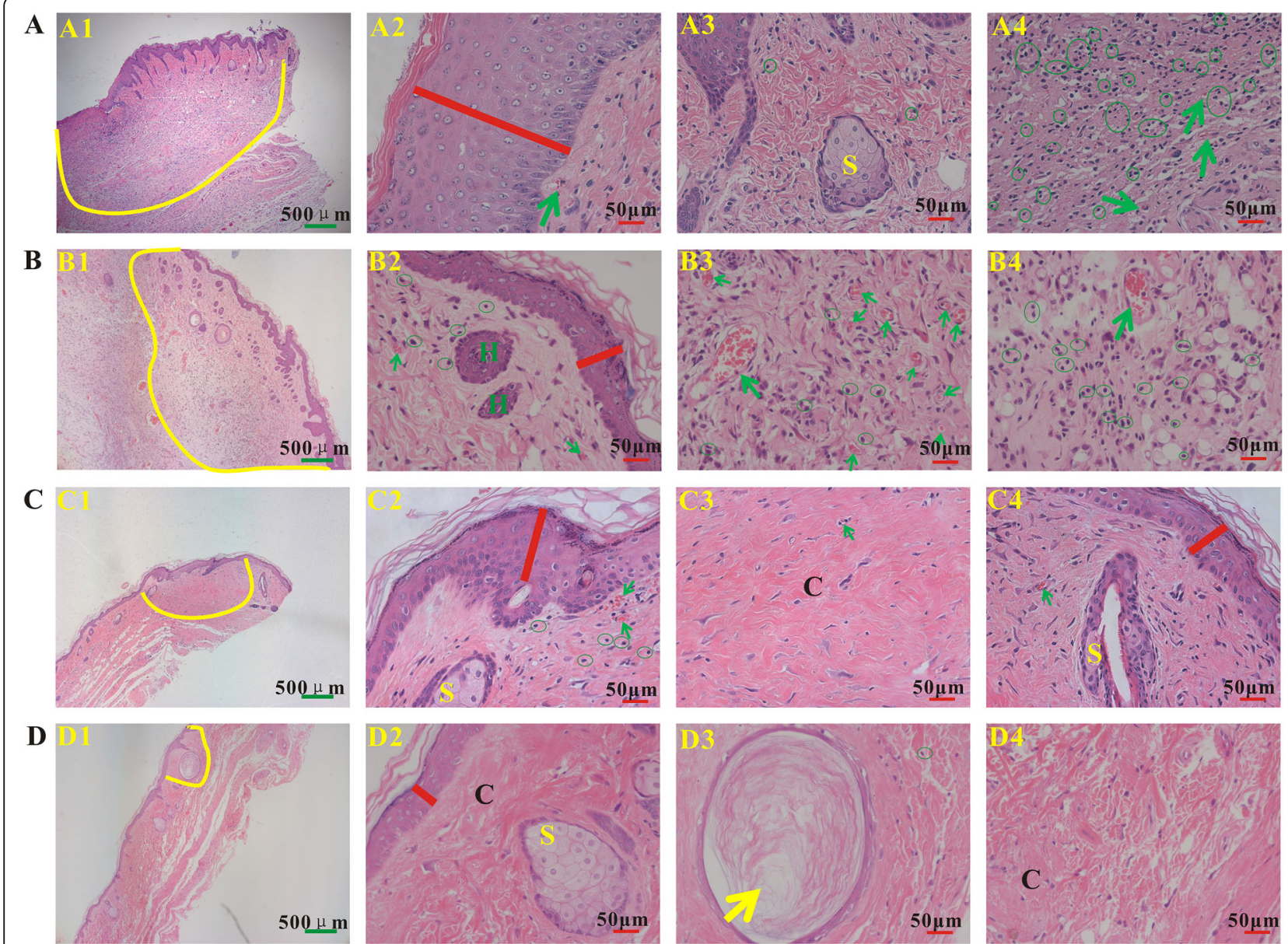

$\mathbf{E}$
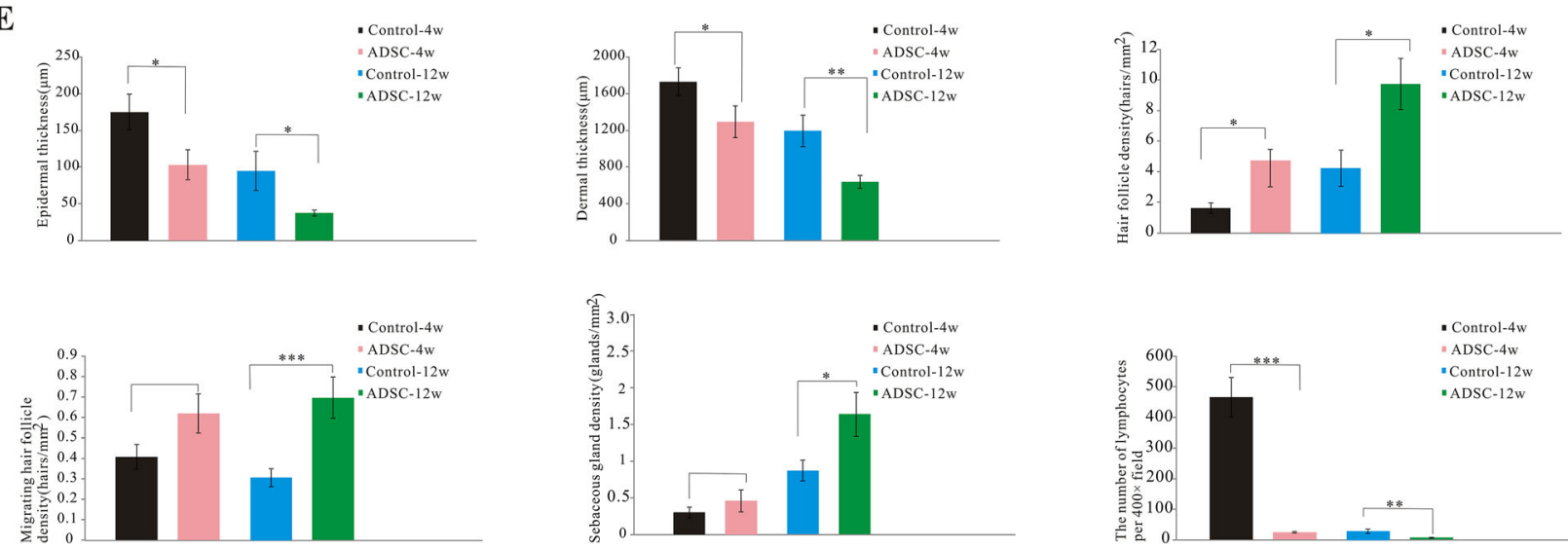

Fig. 3 ADSCs alleviate radiation-induced dermatitis in vivo. Representative images of hematoxylin and eosin-stained skins from rats in the control group at 4 weeks $(\mathbf{A})$ and 12 weeks post-irradiation $(\mathbf{C})$ and the ADSC group at 4 weeks $(\mathbf{B})$ and 12 weeks post-irradiation (D). Yellow curves indicate skin appendage loss. The red line indicates epidermal thickness. The green arrows indicate blood vessels. Green circles mark infiltrated lymphocytes. Statistical analysis of epidermal thickness, dermal thickness, hair follicle density, migrating hair follicle density, sebaceous gland numbers, and lymphocyte number per $400 \times$ field in each group (E) ( $n=8$ /group). The results are expressed as the mean \pm SEM. ${ }^{*} p<0.05,{ }^{* *} p<$ $0.01,{ }^{* *} p<0.001$ vs. the control group. $H$ hair follicle, $S$ sebaceous gland, $C$ collagen

group, whereas the early apoptotic cell proportion did not differ significantly (Fig. 7B). Quantification by flow cytometry showed that ADSC culture supernatant could markedly decrease the total apoptosis ratio. Representative fluorescence photomicrographs of TUNEL-positive apoptotic cells in each 


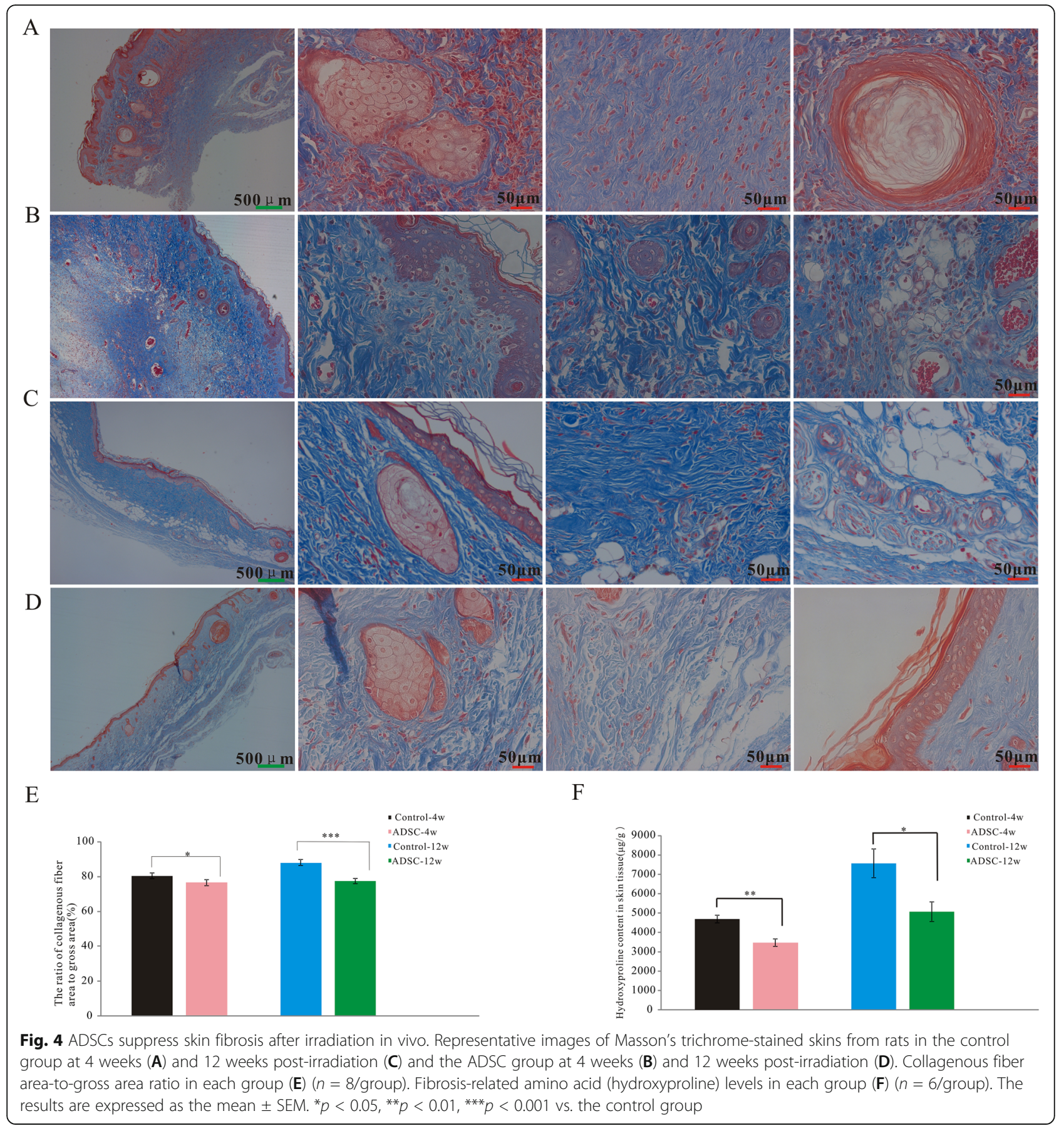

group are shown in Fig. 7A1-3 and 7B1-3. The findings suggested that ADSCs inhibit apoptosis in irradiated cells in vitro.

ADSCs downregulate CTSF expression in vivo and in vitro We evaluated CTSF expression in skin tissues by immunohistochemical staining in vivo (Fig. 8A). At 4 weeks post-irradiation, CTSF expression was increased in the control group, whereas this increase was significantly attenuated in the ADSC group (Fig. 8A, red arrows). At 12 weeks post-irradiation, CTSF was expressed at low levels in the control group, whereas in the ADSC group, CTSF expression was restored to nearly normal levels. Analysis of the average OD value confirmed that CTSF expression was significantly lower in the ADSC group at both 4 and 12 weeks post-irradiation than in the control group at the same time points (Fig. $8 \mathrm{C}$ ).

Next, the expression of CTSF and downstream proapoptotic proteins (Bid, BAX, and caspase 9) and antiapoptotic proteins (Bcl-2 and $\mathrm{Bcl}-\mathrm{XL})$ in skin tissues was 


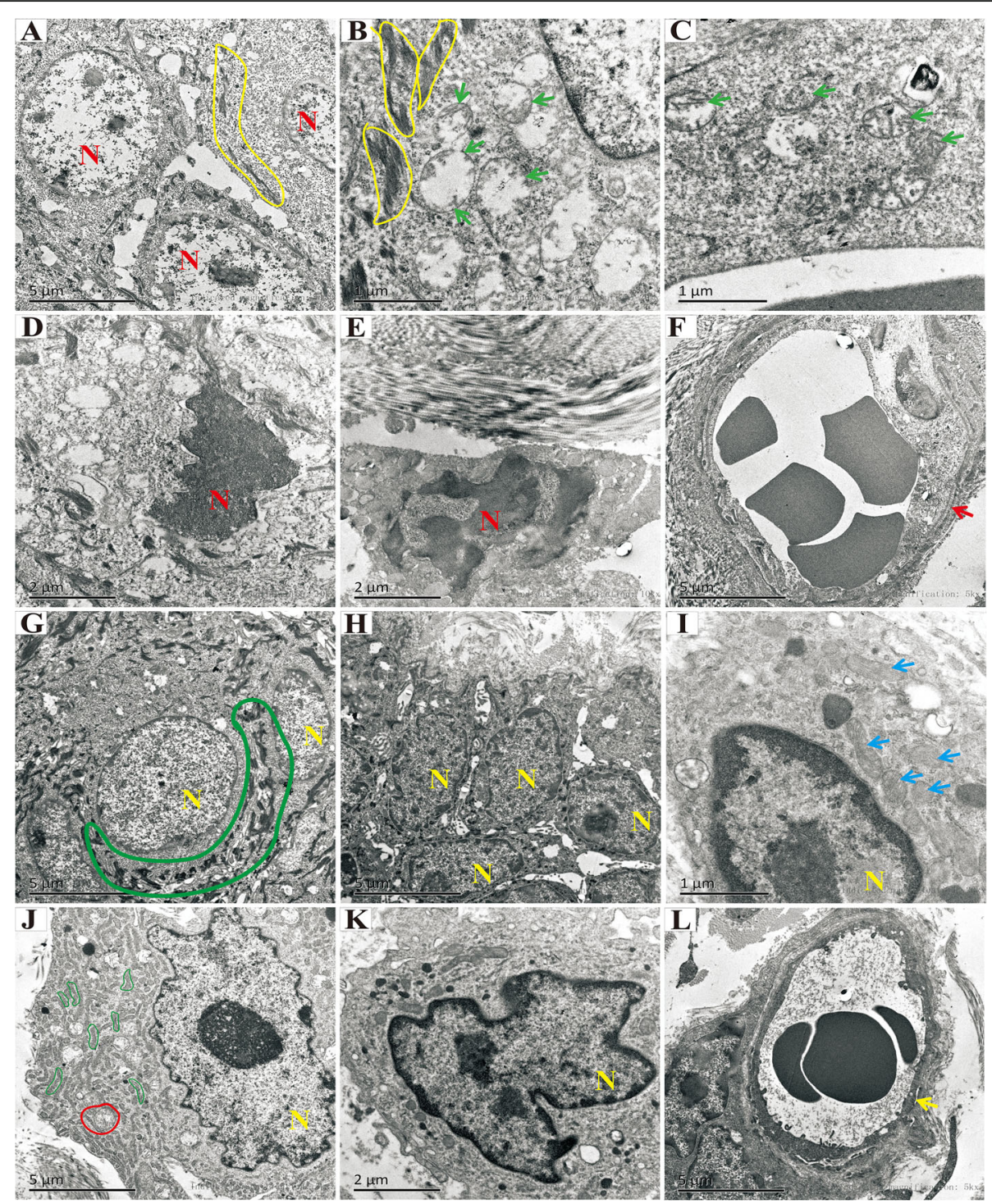

Fig. 5 Transmission electron microscopic analysis of the ultrastructure of skin tissues or rats in the control group at 4 weeks post-irradiation (A-F) and the ADSC group at 4 weeks post-irradiation $(\mathbf{G}-\mathbf{L})$. Yellow curves indicate irregular desmosomes $(\mathbf{A}, \mathbf{B})$. Green arrows indicate damaged mitochondria, with loss of cristae structures (B, C). The red " $\mathrm{N}$ " indicates nuclear pyknosis and dissolution (D-F). Large green curves indicate regular desmosomes $(\mathbf{G})$, and the yellow " $\mathrm{N}$ " indicates regular nuclei in the ADSC group at 4 weeks post-irradiation (G-L). Blue arrows indicate mitochondria with few cristae (I). Small green curves indicate the endoplasmic reticulum (J). Yellow arrows indicate regularly shaped vascular endothelium (L)

detected by western blotting (Fig. 8B). As shown in Fig. $8 \mathrm{D}$, the expression of CTSF, Bid, BAX, and caspase 9 was significantly lower in the ADSC group at both 4 and 12 weeks post-irradiation than in the control group at the same time points. In contrast, the expression of Bcl2 and Bcl-XL was significantly higher in the ADSC group at both 4 and 12 weeks post-irradiation than in the control group at the same time points.

To demonstrate that ADSCs specifically target CTSF, siRNAs were used to knock down CTSF, radiationinduced apoptotic $\mathrm{HaCaT}$ cells were treated with ADSC culture supernatant, the apoptosis was analyzed by flow cytometry (Fig. 9), and the expression of CTSF and downstream proteins was evaluated by western blotting (Fig. 10A,C). As shown in Fig.9, the proportions of $\mathrm{HaCaT}$ cells in total apoptosis were lower in the CTSFsiRNA001 and CTSF-siRNA002 groups than in the NCsiRNA group. As shown in Fig. 10B, the expression of CTSF, BAX, Bid, and caspase 9 was downregulated in the CTSF-siRNA001 and CTSF-siRNA002 groups compared with that in the NC-siRNA group, whereas the expression of $\mathrm{Bcl}-2$ and $\mathrm{Bcl}-\mathrm{XL}$ was upregulated in the CTSF-siRNA001 and CTSF-siRNA002 groups when compared with that in the NC-siRNA group. As shown 


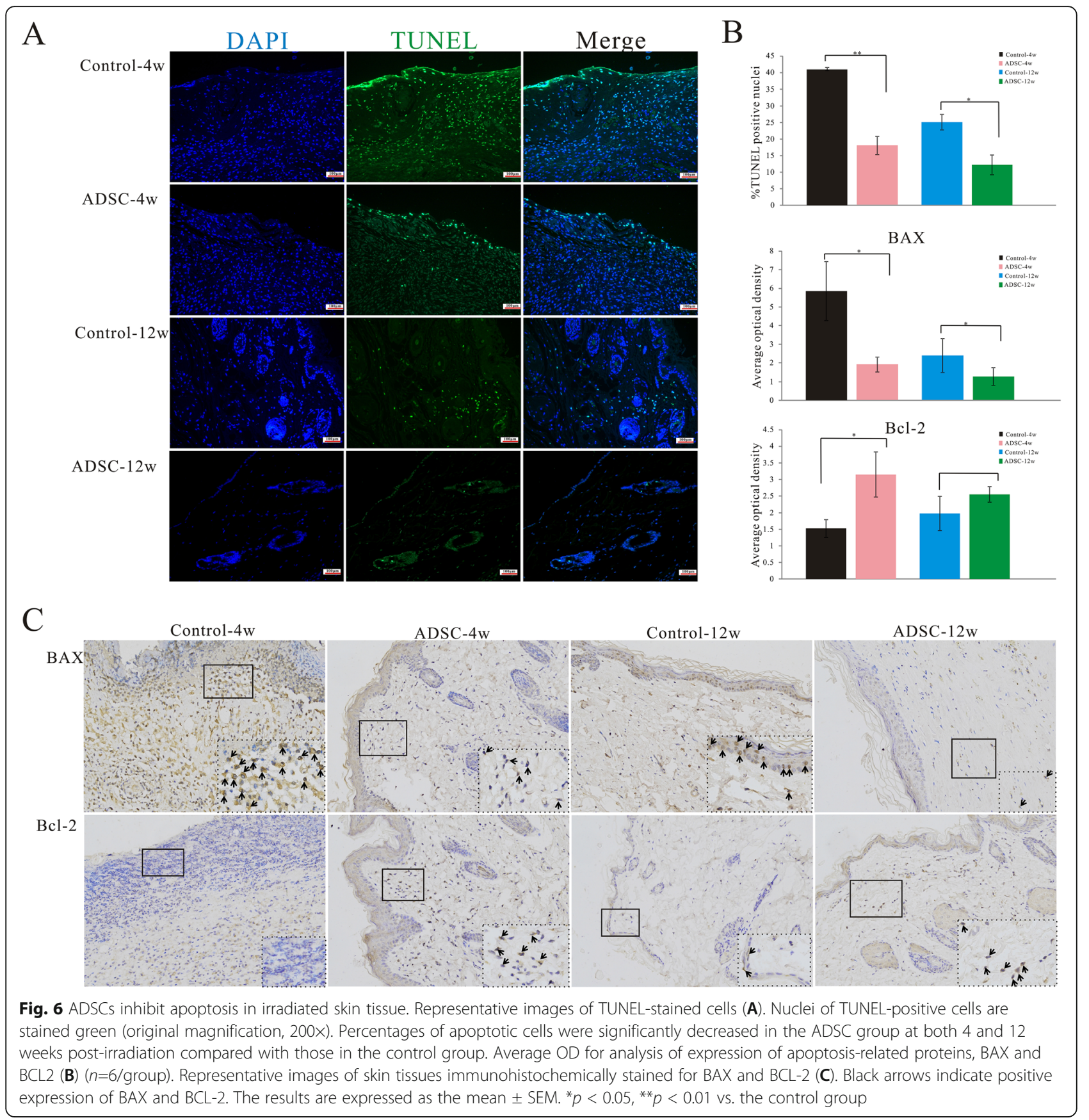

in Fig.10D, in the radiation-induced apoptosis cell model, the expression of CTSF, BAX, Bid, and caspase 9 was downregulated in cells treated with ADSC culture supernatant, CTSF-siRNA001, CTSF-siRNA002, or ADSC culture supernatant plus CTSF-siRNA001 or CTSF-siRNA002 when compared with that in the NCsiRNA group. The expression of Bcl-2 and Bcl-XL was upregulated in cells treated with ADSC culture supernatant, CTSF-siRNA001, CTSF-siRNA002, or ADSC culture supernatant plus CTSF-siRNA001 or CTSFsiRNA002 compared with that in the NC-siRNA group.
These results suggested that ADSCs exhibit an antiapoptotic effect by inhibiting CTSF expression.

\section{Discussion}

In this study, we found that ADSCs decreased skin tissue damage, promoted hair follicle and sebaceous gland regeneration, and inhibited lymphocyte infiltration, apoptosis, and fibrosis formation, demonstrating that ADSCs alleviate acute and chronic radiation-induced dermatitis. Furthermore, ADSCs downregulated cathepsin F expression in a rat model of radiation-induced dermatitis. 

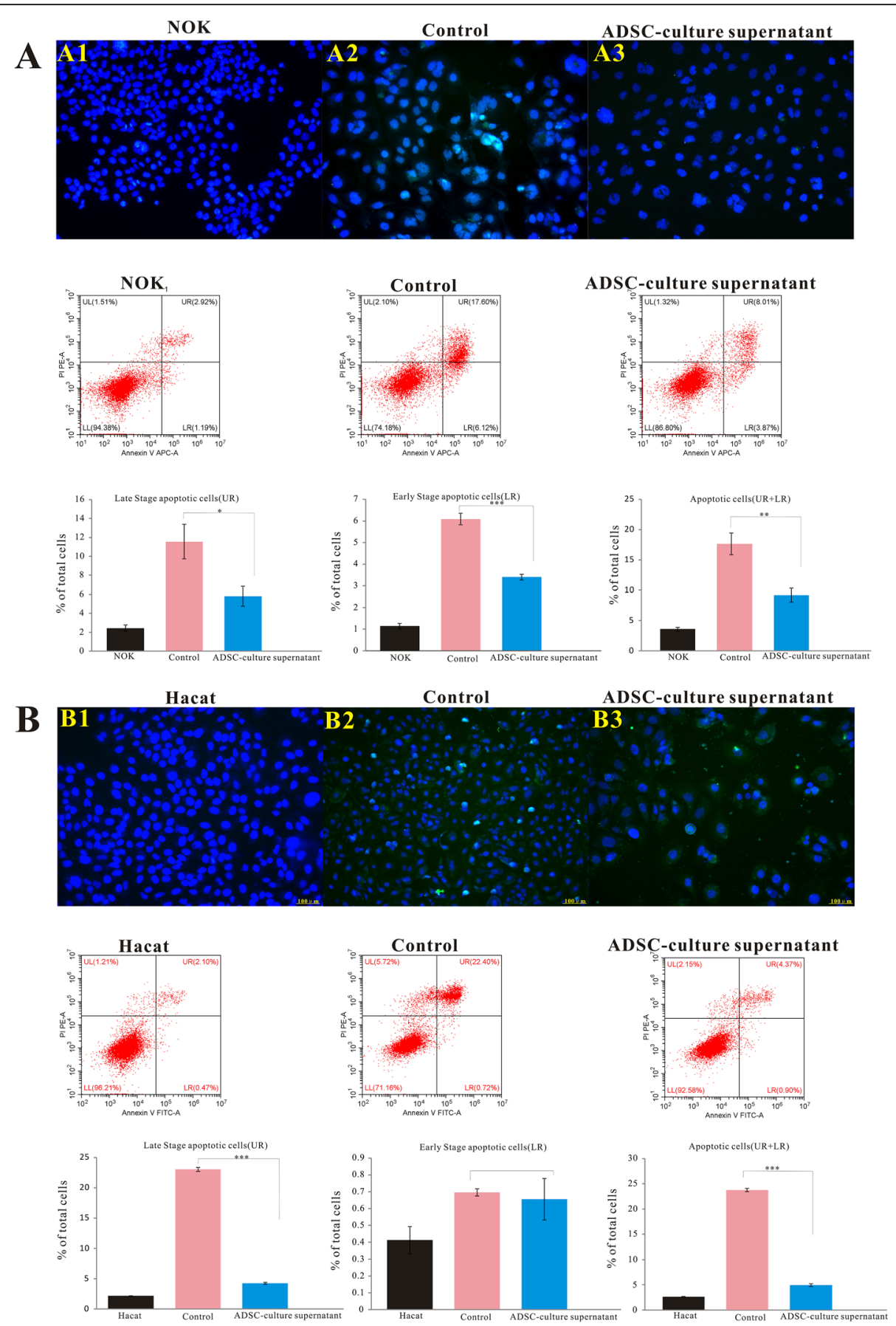

Fig. 7 ADSCs inhibit apoptosis in irradiated epidermal cells in vitro. NOK and HaCaT cells were treated with ADSC culture supernatant for $72 \mathrm{~h}$ and were then harvested for the detection of apoptosis using flow cytometry. Upper left quadrant—necrotic cells; upper right quadrant—late apoptotic cells; lower left quadrant—viable cells; lower right quadrant-early apoptotic cells. Representative fluorescence photomicrographs of TUNEL-positive apoptotic cells are shown $(\mathbf{A}, \mathbf{B})$. The results are expressed as the mean \pm SEM. ${ }^{*} p<0.05,{ }^{* *} p<0.01,{ }^{* * *} p<0.001$ vs. the control group

ADSCs are one of the most promising stem cells as they are relatively easy to harvest and have a high proliferative ability. As ADSCs are not a homogeneous population, there is no unique single surface marker that can be used to characterize them. ADSCs can differentiate into various cell types; adipogenic, osteogenic, and chondrogenic three-line differentiation is the gold standard for characterization. In this study, ADSCs were isolated from inguinal adipose tissues and showed good adipogenic, osteogenic, and chondrogenic differentiation. 


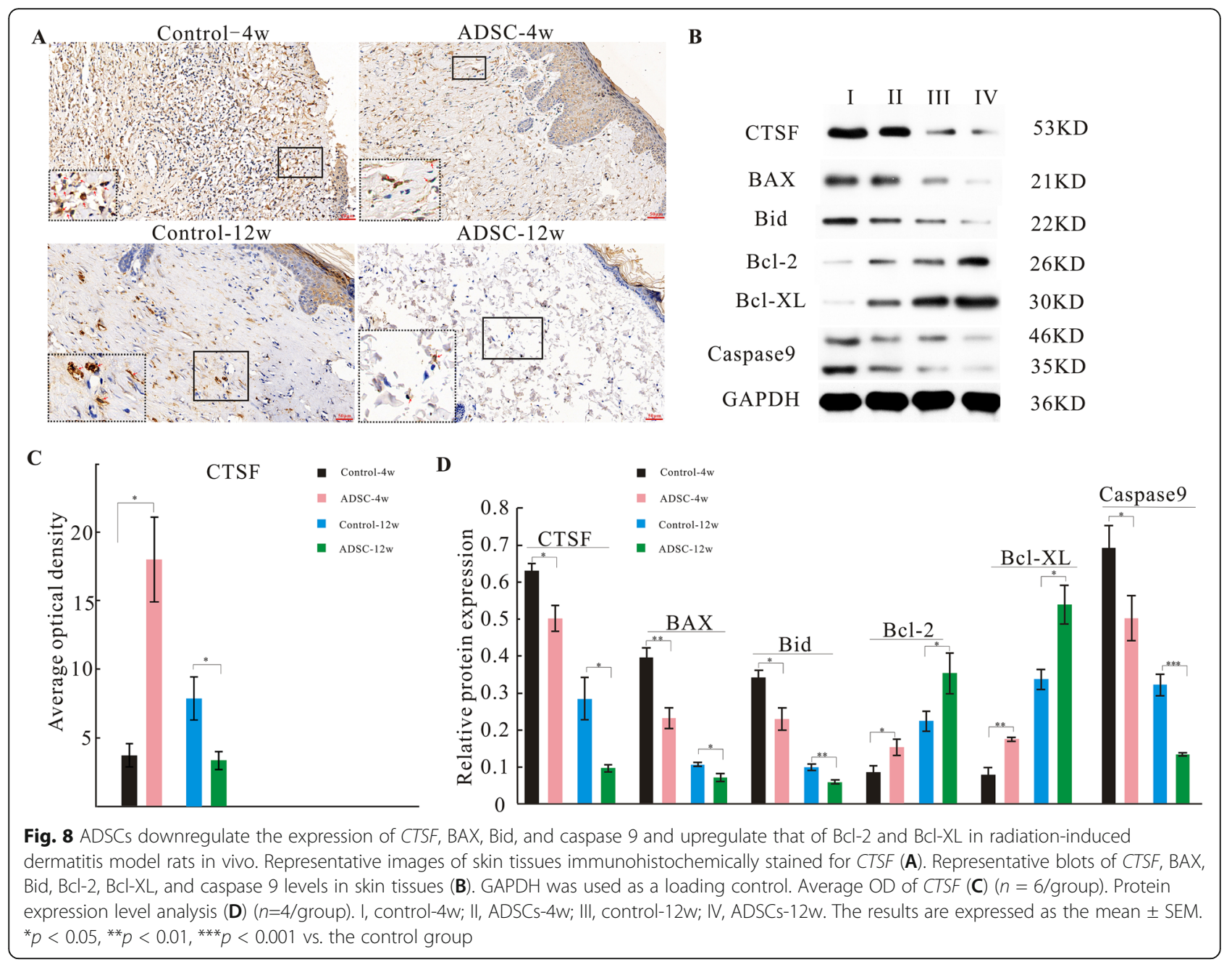

Rigotti et al. reported that the stromal vascular fraction isolated from human lip aspirates alleviated chronic radiation-induced fibrosis clinically in 2005 [24]. Later, Sultan et al. found that fat grafting attenuated acute and chronic radiodermatitis in a murine model [25]. Human mesenchymal stem cells favor healing in the early phase of cutaneous radiation syndrome [26]. Huang et al. found that ADSCs accelerate radiation ulcer healing by increasing angiogenesis [27]. Human umbilical cord mesenchymal stem cells have been shown to improve irradiation-induced skin ulcer healing through keratin generation and epithelial cell proliferation [28]. Human fetal ADSC secretomes accelerated wound healing rate in rats with radiation-induced skin injury by promoting angiogenesis [29]. ADSCs have been shown to have antioxidant activity and protect cells from oxidative injury [30], and $C D 74^{+}$ADSCs have been reported to possess anti-fibrotic activity [31]. Fibrosis is the main feature of chronic radiation-induced dermatitis, and our results showed that ADSCs reduce acute skin injury and fibrosis, which is consistent with previous studies. The data suggest that ADSCs can alleviate acute and chronic radiation-induced dermatitis; however, notably, their effects need to be further enhanced, and we aim to modify ADSCs to improve their effects in future.

Numerous clinical trials using ADSCs have been registered, including studies on their use in the treatment of diabetes, liver disease, fistulas, cardiovascular disease, limb ischemia, graft versus host disease, Crohn's disease, and skin and bone defects. It is necessary to reveal the mechanisms underlying the effects of ADSCs in these pathologies. The ability of ADSCs to repair damaged tissue is mainly attributed to the autocrine and paracrine effects of their secretome, which comprises cytokines, extracellular proteins, and RNAs, including antiinflammatory, immunomodulatory, antioxidant, and regeneration-stimulatory effects. It has been reported that ADSCs stimulate fibroblast and keratinocyte proliferation [32], and exosomes from human ADSCs promote skin fibroblast proliferation and migration [33]. ADSCs promote human umbilical vein endothelial cell 

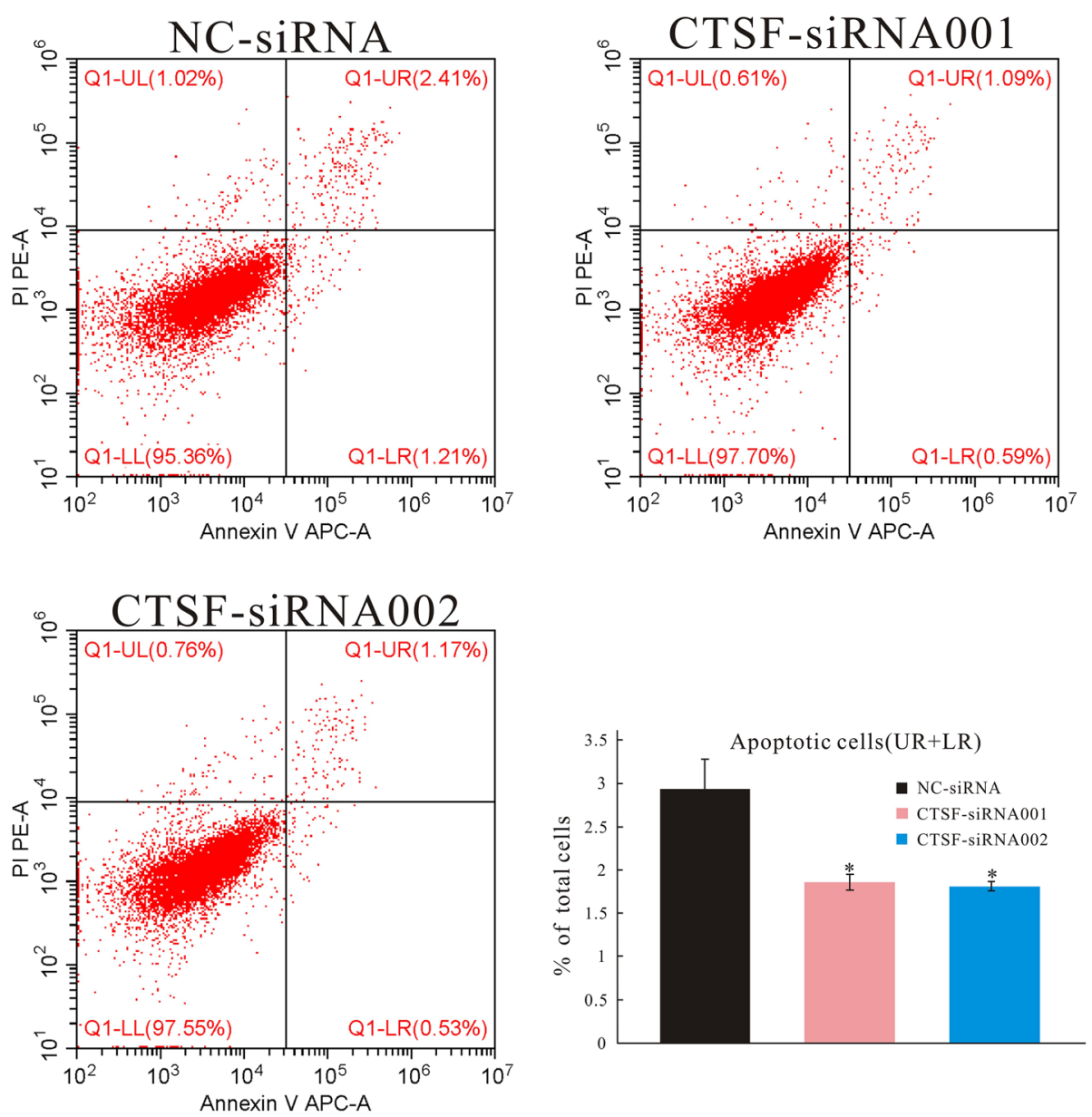

Fig. 9 siRNAs were used to knock down CTSF. HaCaT cells were treated with NC-siRNA, CTSF-siRNA001, and CTSF-siRNA002 and were then harvested for the detection of apoptosis using flow cytometry. Upper left quadrant—necrotic cells; upper right quadrant—late apoptotic cells; lower left quadrant—viable cells; lower right quadrant—early apoptotic cells. The results are expressed as the mean \pm SEM. ${ }^{*} p<0.05$ vs. NC-siRNA group

proliferation, migration, and invasion by releasing miR210 [34]. ADSC-conditioned medium promoted hair growth in vitro, ex vivo, and in vivo [35]. ADSCs can alleviate atopic dermatitis by regulating $B$ cell maturation [36]. Chen et al. found that ADSCs potentially interfere with the formation of silicosis through anti-inflammatory and anti-apoptotic effects [20]. Our previous study showed that inflammation, apoptosis, hair follicle loss, and sebaceous gland loss are the main features of radiation-induced dermatitis in the acute stage [6], and the loss of follicles and sebaceous glands may be due to cell apoptosis. In 2004, Rehman et al. proposed that ADSCs have antiapoptotic potential, which they exert through the secretion of anti-apoptotic factors [37]. To date, the constituents of ADSC-conditioned medium, which is a complex mixture, have not been fully identified. ADSC-secreted anti-apoptotic factors include insulinlike growth factor 1, superoxide dismutase 3, and
miRNA-301a [38]. The exact factors from ADSCs that play roles in alleviating various diseases remain to be clarified. In this study, ADSCs inhibited lymphocyte infiltration and fibrosis formation, promoted hair follicle migration and regeneration, promoted sebaceous gland regeneration, and inhibited apoptosis in vivo and in vitro. In future, we aim to enhance the paracrine ability of ADSCs for anti-fibrosis and proregeneration through modification to improve their efficacy in the treatment of radiation-induced dermatitis. In addition, ADSCs not only downregulated CTSF in vivo and in vitro, but also regulated the expression of CTSF-downstream apoptosis-related proteins, and the change trends of these proteins were consistent with those after CTSF knockdown. These data indicated that ADSCs inhibit apoptosis through downregulating CTSF expression. However, which growth factors or microRNAs secreted from ADSCs directly target CTSF remain to be investigated. 


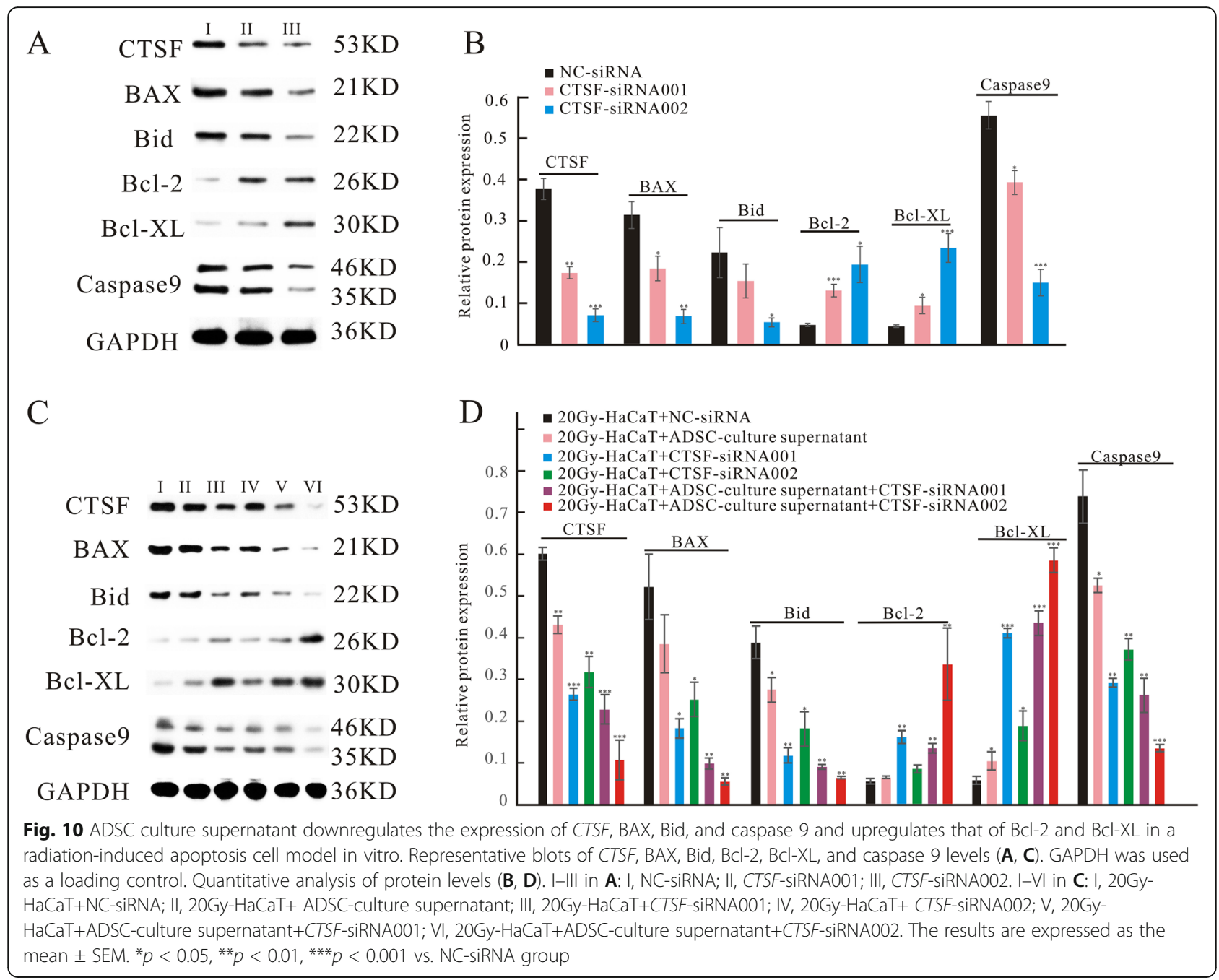

\section{Conclusion}

In summary, we examined the effects of ADSCs on acute and chronic radiation-induced dermatitis in a rat model. We found that ADSCs promote hair follicle and sebaceous gland regeneration and have anti-inflammatory, anti-fibrotic, and anti-apoptotic effects. Further, we found that ADSCs downregulate CTSF and downstream pro-apoptotic proteins (Bid, BAX, and caspase 9) and upregulate downstream anti-apoptotic protein $(\mathrm{Bcl}-2$ and $\mathrm{Bcl}-\mathrm{XL}$ ) expression in vivo and in vitro. ADSCs protect against radiation-induced dermatitis, exhibit an anti-apoptotic effect by inhibiting CTSF expression, and may be a good therapeutic candidate to prevent the development of radiation-induced dermatitis.

\section{Abbreviations}

ADSCs: Adipose-derived stem cells; IL: Interleukin; PBS: Phosphate-buffered saline; DMEM: Dulbecco's modified Eagle's medium; CTSF: Cathepsin F

\section{Acknowledgements}

The authors acknowledge Zichao Ma, Bo Liang, and Chenhui Luo for valuable advice and help; the Animal Laboratory of Hunan Cancer Hospital and The Affiliated Cancer Hospital of Xiangya School of Medicine (Central South University, Changsha, Hunan, China) for providing the animal experimental setup and platform; and the electron microscopy facility at the Institute of Neuroscience, Chinese Academy of Sciences, for assistance with electron microscopy experiments.

\section{Authors' contributions}

CLY performed the experiments, analyzed the data, and edited the manuscript. FYD and YZ performed the experiments and literature search. XWS conceived and designed the study and prepared the manuscript and figure. $X Z$ designed the study and edited the manuscript. All authors read and approved the final manuscript.

\section{Funding}

This study was supported by the National Natural Science Foundation of China (Grant no. 81903262 and no. 82030056) and the Science and Technology Innovation Program of Hunan Province (2020SK51103, 2018SK7005)

Availability of data and materials

The datasets used and/or analyzed during the current study are available from the corresponding author on reasonable request. 


\section{Declarations}

\section{Ethics approval and consent to participate}

Ethics approval was obtained from the Animal Ethics Committee of Hunan Cancer Hospital, the Affiliated Cancer Hospital of Xiangya School of Medicine, Central South University.

\section{Consent for publication}

Not applicable

\section{Competing interests}

The authors declare that they have no competing interests.

\section{Author details}

'Department of Head and Neck Surgery, Central Laboratory, Hunan Cancer Hospital and The Affiliated Cancer Hospital of Xiangya School of Medicine, Tongzipo Road 283, Changsha 410013, Hunan Province, China. ${ }^{2}$ Department of Radiation Oncology, Hunan Cancer Hospital and The Affiliated Cancer Hospital of Xiangya School of Medicine, Central South University, Tongzipo Road 283, Changsha 410013, Hunan Province, China. ${ }^{3}$ Department of Radiotherapy, National Cancer Center/National Clinical Research Center for Cancer/Cancer Hospital \& Shenzhen Hospital, Chinese Academy of Medical Sciences and Peking Union Medical College, Longgang District Baohe Avenue Road 113, Shenzhen 518116, Guangdong Province, China. ${ }^{4}$ Nursing Department, Hunan Cancer Hospital and The Affiliated Cancer Hospital of Xiangya School of Medicine, Tongzipo Road 283, Changsha 410013, Hunan Province, China. ${ }^{5}$ Institute of Neuroscience, Chinese Academy of Sciences, 320, Yue Yang Road, Shanghai 200031, China. ${ }^{6}$ Pathology Department, Hunan Cancer Hospital and The Affiliated Cancer Hospital of Xiangya School of Medicine, Tongzipo Road 283, Changsha 410013, Hunan Province, China. ${ }^{7}$ Institute of Rocket Force Medicine, State Key Laboratory of Trauma, Burns and Combined Injury, Third Military Medical University, No. 30, Main Street, Gaotanyan, Shapingba District, Chongqing 400038, China.

Received: 21 January 2021 Accepted: 14 July 2021

\section{Published online: 09 August 2021}

\section{References}

1. Hegedus F, Mathew LM, Schwartz RA. Radiation dermatitis: an overview. Int J Dermatol. 2017;56(9):909-14. https://doi.org/10.1111/ijd.13371.

2. Ryan JL. Ionizing radiation: the good, the bad, and the ugly. J Invest Dermatol. 2012;132(3 Pt 2):985-93. https://doi.org/10.1038/jid.2011.411.

3. Harper $J \mathrm{~L}$, Franklin LE, Jenrette JM, Aguero EG. Skin toxicity during breast irradiation: pathophysiology and management. South Med J. 2004:97(10): 989-93. https://doi.org/10.1097/01.SMJ.0000140866.97278.87.

4. Wong RK, Bensadoun RJ, Boers-Doets CB, et al. Clinical practice guidelines for the prevention and treatment of acute and late radiation reactions from the MASCC Skin Toxicity Study Group. Support Care Cancer. 2013;21(10): 2933-48. https://doi.org/10.1007/s00520-013-1896-2.

5. Hashimoto I, Sedo H, Inatsugi K, Nakanishi H, Arase S. Severe radiationinduced injury after cardiac catheter ablation: a case requiring free anterolateral thigh flap and vastus lateralis muscle flap reconstruction on the upper arm. J Plast Reconstr Aesthet Surg. 2008;61(6):704-8. https://doi. org/10.1016/j.bjps.2007.01.003.

6. Sheng $X$, Zhou $Y$, Wang $H$, Shen $Y$, Liao Q, Rao Z, et al. Establishment and characterization of a radiation-induced dermatitis rat model. J Cell Mol Med. 2019;23(5):3178-89. https://doi.org/10.1111/jcmm.14174.

7. Huen MS, Chen J. The DNA damage response pathways: at the crossroad of protein modifications. Cell Res. 2008;18(1):8-16. https://doi.org/10.1038/cr.2 007.109

8. Coates PJ, Appleyard MV, Murray K, et al. Differential contextual responses of normal human breast epithelium to ionizing radiation in a mouse xenograft model. Cancer Res. 2010;70(23):9808-15. https:/doi.org/10.1158/ 0008-5472.CAN-10-1118.

9. Tak JK, Park JW. The use of ebselen for radioprotection in cultured cells and mice. Free Radic Biol Med. 2009;46(8):1177-85. https://doi.org/10.1016/j. freeradbiomed.2009.01.023

10. Gandhi S, Chandna S. Radiation-induced inflammatory cascade and its reverberating crosstalks as potential cause of post-radiotherapy second malignancies. Cancer Metastasis Rev. 2017;36(2):375-93. https://doi.org/10.1 007/s10555-017-9669-x
11. Calveley VL, Khan MA, Yeung IW, et al. Partial volume rat lung irradiation: temporal fluctuations of in-field and out-of-field DNA damage and inflammatory cytokines following irradiation. Int J Radiat Biol. 2005;81(12): 887-99. https://doi.org/10.1080/09553000600568002.

12. Ezquerro S, Mocha F, Fruhbeck G, et al. Ghrelin Reduces TNF-alpha-Induced Human Hepatocyte Apoptosis, Autophagy, and Pyroptosis: Role in ObesityAssociated NAFLD. J Clin Endocrinol Metab. 2019;104(1):21-37. https://doi. org/10.1210/jc.2018-01171.

13. Gao C, Fu Q, Su B, Song H, Zhou S, Tan F, et al. The involvement of cathepsin $\mathrm{F}$ gene (CTSF) in turbot (Scophthalmus maximus L.) mucosal immunity. Fish Shellfish Immunol. 2017;66:270-9. https://doi.org/10.1016/j. fsi.2017.05.030.

14. Ji C, Zhao Y, Kou YW, Shao H, Guo L, Bao CH, et al. Cathepsin F Knockdown Induces Proliferation and Inhibits Apoptosis in Gastric Cancer Cells. Oncol Res. 2018;26(1):83-93. https://doi.org/10.3727/096504017X14928634401204.

15. Janic A, Valente LJ, Wakefield MJ, di Stefano L, Milla L, Wilcox S, et al. DNA repair processes are critical mediators of p53-dependent tumor suppression. Nat Med. 2018;24(7):947-53. https://doi.org/10.1038/s41591018-0043-5.

16. Zuk PA, Zhu M, Mizuno H, Huang J, Futrell JW, Katz AJ, et al. Multilineage cells from human adipose tissue: implications for cell-based therapies. Tissue Eng. 2001;7(2):211-28. https://doi.org/10.1089/107632701300062859.

17. Shukla L, Morrison WA, Shayan R. Adipose-derived stem cells in radiotherapy injury: a new frontier. Front Surg. 2015;2:1.

18. Lee M, Ban JJ, Yang S, et al. The exosome of adipose-derived stem cells reduces beta-amyloid pathology and apoptosis of neuronal cells derived from the transgenic mouse model of Alzheimer's disease. Brain Res. 1691; 2018:87-93.

19. Kotani T, Masutani R, Suzuka T, Oda K, Makino S, li M. Anti-inflammatory and anti-fibrotic effects of intravenous adipose-derived stem cell transplantation in a mouse model of bleomycin-induced interstitial pneumonia. Sci Rep. 2017:7(1):14608. https://doi.org/10.1038/s41598-017-15022-3.

20. Chen S, Cui G, Peng C, Lavin MF, Sun X, Zhang E, et al. Transplantation of adipose-derived mesenchymal stem cells attenuates pulmonary fibrosis of silicosis via anti-inflammatory and anti-apoptosis effects in rats. Stem Cell Res Ther. 2018;9(1):110. https://doi.org/10.1186/s13287-018-0846-9.

21. Liu Z, Rao Z, Sheng X, Long Y, Zhou X. Effect of adipose-derived stem cells on radiation-induced acute skin injury in rats. Zhong Nan Da Xue Xue Bao Yi Xue Ban. 2019;44(2):150-7. https://doi.org/10.11817/j.issn.1672-7347.2019. 02.006 .

22. Field SB, Law MP. The relationship between early and late radiation damage in rodents' skin. Int J Radiat Biol Relat Stud Phys Chem Med. 1976;30(6):55764. https://doi.org/10.1080/09553007614551411.

23. Kumar S, Kolozsvary A, Kohl R, Lu M, Brown S, Kim JH. Radiation-induced skin injury in the animal model of scleroderma: implications for postradiotherapy fibrosis. Radiat Oncol. 2008;3(1):40. https://doi.org/10.1186/174 8-717X-3-40.

24. Rigotti G, Marchi A, Galie M, et al. Clinical treatment of radiotherapy tissue damage by lipoaspirate transplant: a healing process mediated by adiposederived adult stem cells. Plast Reconstr Surg. 2007;119(5):1409-22 discussion 1423-1404 https://doi.org/10.1097/01 prs.000025604747909.71.

25. Sultan SM, Stern CS, Allen RJ Jr, Thanik VD, Chang CC, Nguyen PD, et al. Human fat grafting alleviates radiation skin damage in a murine model. Plast Reconstr Surg. 2011;128(2):363-72. https://doi.org/10.1097/PRS.0b013 e31821e6e90.

26. Francois S, Mouiseddine M, Mathieu N, et al. Human mesenchymal stem cells favour healing of the cutaneous radiation syndrome in a xenogenic transplant model. Ann Hematol. 2007:86(1):1-8. https://doi.org/10.1007/s002 77-006-0166-5

27. Huang SP, Huang $\mathrm{CH}$, Shyu JF, Lee HS, Chen SG, Chan JYH, et al. Promotion of wound healing using adipose-derived stem cells in radiation ulcer of a rat model. J Biomed Sci. 2013:20(1):51. https://doi.org/10.1186/14230127-20-51

28. Liu Z, Yu D, Xu J, Li X, Wang X, He Z, et al. Human umbilical cord mesenchymal stem cells improve irradiation-induced skin ulcers healing of rat models. Biomed Pharmacother. 2018;101:729-36. https://doi.org/10.1016/ j.biopha.2018.02.093

29. Rong $X, L i J$, Yang $Y$, Shi L, Jiang T. Human fetal skin-derived stem cell secretome enhances radiation-induced skin injury therapeutic effects by promoting angiogenesis. Stem Cell Res Ther. 2019;10(1):383. https://doi. org/10.1186/s13287-019-1456-X. 
30. Kim WS, Park BS, Kim HK, Park JS, Kim KJ, Choi JS, et al. Evidence supporting antioxidant action of adipose-derived stem cells: protection of human dermal fibroblasts from oxidative stress. J Dermatol Sci. 2008;49(2):133-42. https://doi.org/10.1016/j.jdermsci.2007.08.004.

31. Borrelli MR, Patel RA, Adem S, Diaz Deleon NM, Shen AH, Sokol J, et al. The antifibrotic adipose-derived stromal cell: Grafted fat enriched with CD74+ adipose-derived stromal cells reduces chronic radiation-induced skin fibrosis. Stem Cells Transl Med. 2020;9(11):1401-13. https://doi.org/10.1002/ sctm.19-0317.

32. Neri S, Bourin P, Peyrafitte JA, Cattini L, Facchini A, Mariani E. Human adipose stromal cells (ASC) for the regeneration of injured cartilage display genetic stability after in vitro culture expansion. PLoS One. 2013;8(10): e77895. https://doi.org/10.1371/journal.pone.0077895.

33. Choi EW, Seo MK, Woo EY, Kim SH, Park EJ, Kim S. Exosomes from human adipose-derived stem cells promote proliferation and migration of skin fibroblasts. Exp Dermatol. 2018;27(10):1170-2. https://doi.org/10.1111/ exd.13451.

34. Zheng Z, Liu L, Zhan Y, Yu S, Kang T. Adipose-derived stem cell-derived microvesicle-released miR-210 promoted proliferation, migration and invasion of endothelial cells by regulating RUNX3. Cell Cycle. 2018;17(8): 1026-33. https://doi.org/10.1080/15384101.2018.1480207.

35. Won CH, Park GH, Wu X, Tran TN, Park KY, Park BS, et al. The Basic Mechanism of Hair Growth Stimulation by Adipose-derived Stem Cells and Their Secretory Factors. Curr Stem Cell Res Ther. 2017;12(7):535-43. https:/ doi.org/10.2174/1574888X12666170829161058.

36. Shin TH, Lee BC, Choi SW, Shin JH, Kang I, Lee JY, et al. Human adipose tissue-derived mesenchymal stem cells alleviate atopic dermatitis via regulation of B lymphocyte maturation. Oncotarget. 2017;8(1):512-22. https://doi.org/10.18632/oncotarget.13473.

37. Rehman J, Traktuev D, Li J, Merfeld-Clauss S, Temm-Grove CJ, Bovenkerk JE, et al. Secretion of angiogenic and antiapoptotic factors by human adipose stromal cells. Circulation. 2004;109(10):1292-8. https://doi.org/10.1161/01.CIR. 0000121425.42966.F1.

38. Li X, Ma T, Sun J, Shen M, Xue X, Chen Y, et al. Harnessing the secretome of adipose-derived stem cells in the treatment of ischemic heart diseases. Stem Cell Res Ther. 2019;10(1):196. https://doi.org/10.1186/s13287-019-1289-7.

\section{Publisher's Note}

Springer Nature remains neutral with regard to jurisdictional claims in published maps and institutional affiliations.

Ready to submit your research? Choose BMC and benefit from:

- fast, convenient online submission

- thorough peer review by experienced researchers in your field

- rapid publication on acceptance

- support for research data, including large and complex data types

- gold Open Access which fosters wider collaboration and increased citations

- maximum visibility for your research: over $100 \mathrm{M}$ website views per year

At $\mathrm{BMC}$, research is always in progress.

Learn more biomedcentral.com/submissions 